\title{
Multiple Representations in 8th Grade Mathematics Textbook and the Extent to which Teachers Implement Them
}

\author{
Mohammad Alkhateeb ${ }^{1^{*}}$
}

\author{
${ }^{1}$ Hashemite University, JORDAN \\ *CORRESPONDENCE: $\square$ mkm7879@yahoo.com
}

\begin{abstract}
This study, firstly, aimed at investigating the various types of the multiple math representations and transitions among them in the $8^{\text {th }}$ grade math textbook, and secondly, teachers' use of these representations in teaching. The current study employed the content analysis approach to investigate the multiple mathematical representations and transitions among them in 8th Grade Mathematics Textbook. An observation method was used to analyses the teacher practices ( $n=35$ observations), and record the representations and transitions. The results showed that there was an existence of symbol and verbal representations in the textbook and teachers' implementation. Meanwhile, the other three representations (pictures and figures, models and Cutters, and life situations) and the transactions between them were at low rates in the textbook and teachers' implementation.
\end{abstract}

Keywords: symbol representation, verbal representation, picture representation, model representation, life situation representation, teachers' practices

\section{INTRODUCTION}

Many international researchers who are interested in mathematics such as (Cai \& Lester, 2005; Dreher \& Kuntze, 2015; Dwi, Subanji, Hidayanto \& Anwar, 2017; You \& Quinn, 2010) consider mathematical representations as the basic feature to teach and learn math, whereas researchers mentioned that mathematical representations are closely improving students' learning and understanding of math (Anthony \& Walshaw, 2009; Greeno \& Hall, 1997; Santulli, 2009; Tripathi, 2008). Nonetheless, there are only few studies that highlighted the role of the textbook and teachers in facilitating students' understanding through using mathematical representations (Bal, 2015; Bayazit, 2011; Yan \& Lianghuo, 2006; Ulu, 2017)

Reviewing the literature concerning mathematical representations; the work of Lesh, Post, and Behr (1987) was one of the most important in this area, they constructed a model of the various elements in which mathematical representations are structured. This model is consisted of five elements (Figure 1); (1) representations by written symbols such as writing numbers or symbols to express the math concept, (2) spoken representations which indicate expressing the math concept by words which allows often for more rephrasing the words and linking the concepts with other areas and life situations. (3) Experience based interpreting representations by real-life context which use the daily life events that fit the math concept, (4) manipulative representations are using any tool that student can hold by hand such as models and cutters. Finally (5) representations by pictures or diagrams include pictures, drawings, and tables.

Article History: Received 8 October $2018 \bullet$ Revised 23 November $2018 \bullet$ Accepted 23 November 2018

(C) 2019 by the authors; licensee Modestum Ltd., UK. Open Access terms of the Creative Commons Attribution 4.0 International License (http://creativecommons.org/licenses/by/4.0/) apply. The license permits unrestricted use, distribution, and reproduction in any medium, on the condition that users give exact credit to the original author(s) and the source, provide a link to the Creative Commons license, and indicate if they made any changes. 


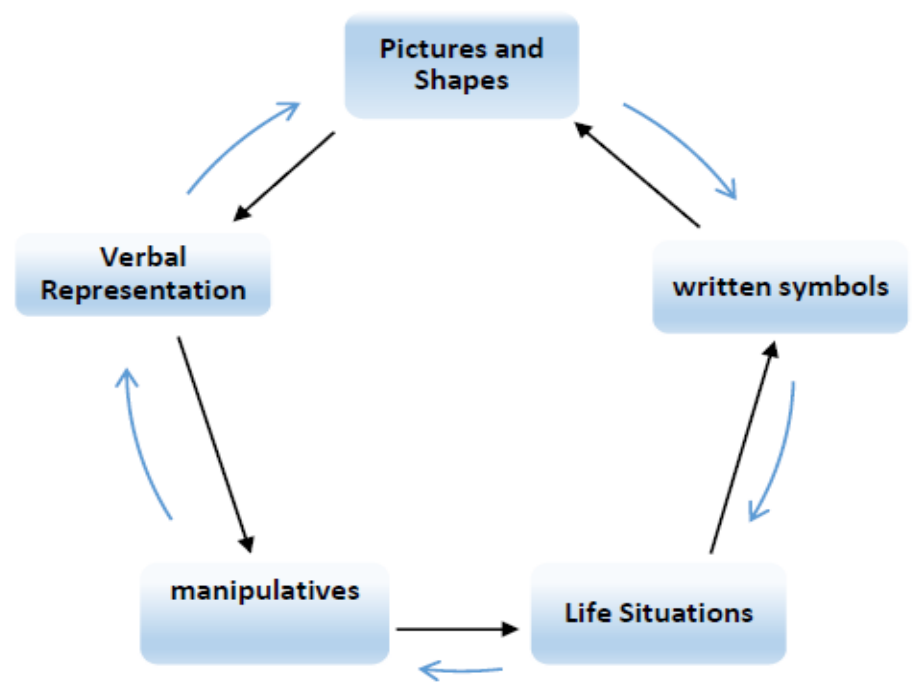

Figure 1. Lesh, Post \& Behr Model for the Math Representations

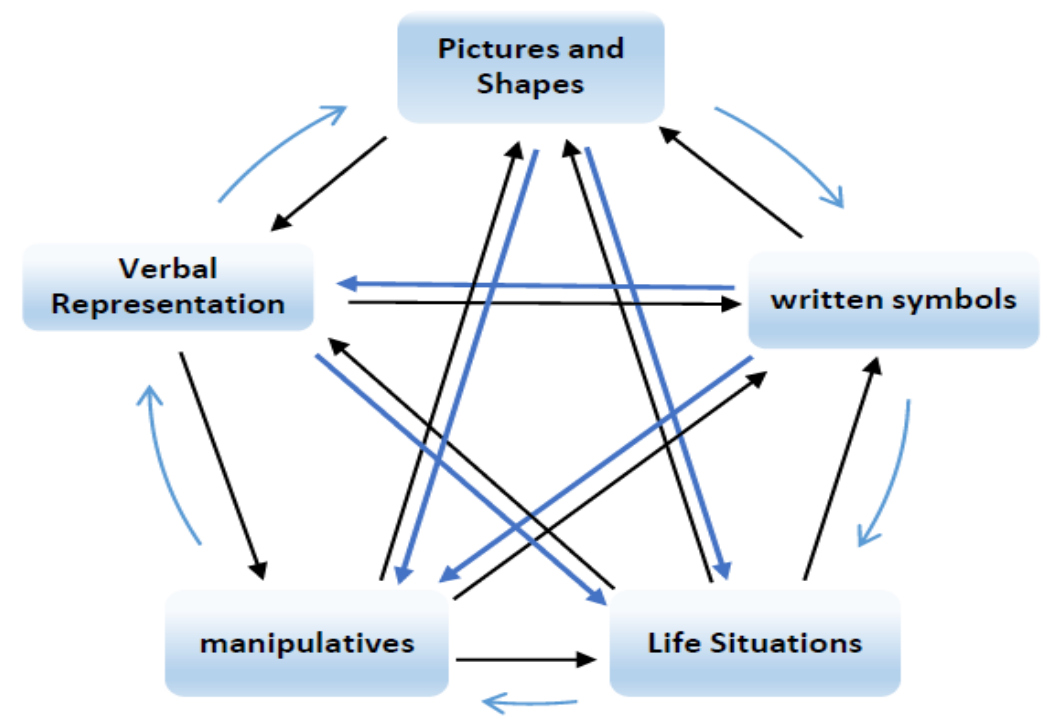

Figure 2. Model of Lesh et al. (2003) for the Math Representations

Moreover, the previous model of Lesh and his other researchers has been improved by Lesh, Cramer, Doerr, Post and Zawojewski (2003) - Figure 2- to construct a network among the five elements and the interactions among them.

The improved network showed that in math learning, it is more efficient to make transitions among the five elements according to students' developmental levels and understanding; moreover, the transitions among the five elements help teachers to transform the concepts of math into abstract meanings, which in turn allow teachers to make changes in their strategies and teaching methods to improve the quality of their instructional approach (Doerr \& Lesh, 2011; Koretsky, Keeler, Ivanovitch \& Cao, 2018; Lesh, \& Lehrer, 2003; Lesh, \& Harel, 2003).

Gagatsis and Shikalli (2004) mentioned that the more teachers use representations and their multiple transitions in their teaching, the more likely students able to understand and success in math. Moreover, Anthony and Walshaw (2009) while they reviewed many studies concerning representations and transitions found that the presentations link between different math ideas and concepts, which encourage students to understand the different connections among the concepts through discovering different representations of the concept. Hwang, Chen, Dung and Yang (2007) indicated that the improving students' skills in using multiple 
math representations are the key of their success in solving the math problems. Bayazit (2011) showed that most of the participants depend on the symbols representation of conjugation; and that the students' alternation between the iconic and algebraic representation of conjugation seems an important stage for developing the conjugation concept.

Therefore, this current study presents further evidence for that connection between math textbook and teachers and students' understanding of math. The theoretical framework depends on the model which was constructed by Lesh et al. (2003) for the math representations, as content analysis approach was used to explore the use of presentations and to investigate the various transitions in the textbook of 8th grade specifically the algebra unit which. Moreover, the significance of the current study also emerges from the use of qualitative method using observation tool to examine teacher's use of representations while teaching algebra unit and the extent to which they apply representations transitions according to Lesh et al. (2003) model.

\section{RESEARCH METHODOLOGY}

The current study employed the content analysis approach to investigate the multiple mathematical representations and transitions among them in the textbook of 8th grade due to the importance of the eighth grade as the Ministry of Education in Jordan deems a stage through which it can enable students assimilate the different math concepts. Furthermore, the TIMSS exams are made on the grade, because there is a similarity in the content of the Jordanian, European, and American mathematics textbooks for the eighth grade in the fields of numbers, geometry, algebra, statistics and probabilities, as they are all built in light of the NCTM. The researcher argues that content analysis will provide a clear image of multiple mathematical representations and transitions among them as it illustrated the data in details.

In addition, the observation tool was also used to examine the extent of the math teachers' use of these representations and transitions in their classes. The researcher visited the Zarqa educational district, where he was told that it is divided into five areas. He asked for one teacher from each area for his observation that has to have BA degree in Maths and teach 8th grade classes for at least 5 years. After having the names of teachers, the researcher contacted them personally to ask them for their agreement on participation. Three male teachers and two female teachers were the chosen participants who were coded by their first letter of their names and took numbers from 1-5. Focusing more narrowly, this study addresses the following questions:

1- What are the distributions of Lesh's network model of representations and their transitions in the unit of Algebra in the textbook of 8th grade?

2- To which extent teachers use representations and transitions among them while teaching the unit of Algebra in the textbook of 8 th grade?

\section{Reliability and Validity}

The researcher developed a table that contained all the determined variables of the five elements of Lesh's network model and their transitions, and then the research asked other two colleges to develop a table to the same topic. The consistency of the data obtained created the basic for ensuring validity and reliability. A total of 7 lessons and 7 class visits were accessed by October 20th 2017 and which have been analyzed individually by the three researchers who used the prepared table. Namely, representation in written symbols (R), verbal representation $(\mathrm{L})$, real life situation representations $(\mathrm{H})$, manipulative representation $(\mathrm{N})$, and pictures and diagrams representation (S). The tables were compared to identify the differences among the related representations and their transitions and which were examined again.

Moreover, the comparison allowed obtaining common themes, which strengthened the validity and reliability of the table that was used in this study. Finally, for ensuring the interpretative validity, the researcher let other math supervisor's members who supervise teachers to read the initial findings to give any feedback of incorrect representations.

\section{Procedures}

1- Analysis the algebra unit in eighth grade math textbook, calculation of the percentages of the representations, as well as the transition amount among the applied representations.

2- Attend all lessons on the topic of algebra for every participant, calculation of the percentage of each of the math representations and transitions among them. Thereafter, comparing them with textbook representations and transitions, on one hand, and with Lesh et al. (2003) model on the other, as an 
Table 1. The Frequencies and Percentages of the Distribution of representations in math. Textbook

\begin{tabular}{cccc}
\hline representations & frequencies & percentages & distribution \\
\hline $\mathrm{R}$ & 144 & 38.50 & medium \\
\hline $\mathrm{L}$ & 143 & 38.24 & medium \\
\hline $\mathrm{H}$ & 36 & 9.63 & very low \\
\hline $\mathrm{N}$ & 11 & 2.94 & very low \\
\hline $\mathrm{S}$ & 40 & 10.70 & very low \\
\hline Total & 374 & & \\
\hline
\end{tabular}

Table 2. The transitions among the multiple representations in math. Textbook and its percentages

\begin{tabular}{ccccc}
\hline \multicolumn{2}{c}{ Translations } & frequencies & percentages & Distribution \\
\hline $\mathrm{R} \longrightarrow \mathrm{L}$ & 140 & 29 & low \\
$\mathrm{L} \longrightarrow \mathrm{R}$ & 116 & 24 & low \\
\hline $\mathrm{S} \longrightarrow \mathrm{R}$ & 92 & 19 & very low \\
\hline $\mathrm{L} \longrightarrow \mathrm{S}$ & 43 & 9 & very low \\
\hline $\mathrm{R} \longrightarrow \mathrm{S}$ & 19 & 4 & very low \\
\hline $\mathrm{S} \longrightarrow \mathrm{N}$ & 19 & 4 & very low \\
\hline $\mathrm{S} \longrightarrow \mathrm{L}$ & 5 & 1 & very low \\
\hline Other translations & 51 & 2 & very low \\
\hline \multicolumn{2}{c}{ total } & 485 & &
\end{tabular}

attempt to link between what is theoretically stated in the textbook and what is going inside the classroom.

There are some certain limitations to this study: The current study used a specific method of qualitative research - content analysis- which categorizes the raw data by developing a descriptive data such as percentages and frequencies to reach a conclusion, and this could limit the comparison with other qualitative research methods. Moreover, this study examined the algebra unit in the $8^{\text {th }}$ standardized textbook grade in Jordan the thing which may influence the applicable features in other contexts, therefore, generalization of the findings will not be encouraged to make.

\section{FINDINGS}

Q1-What are the distributions of Lesh's network model of representations and their transitions in the unit of Algebra in the textbook of $8^{\text {th }}$ grade?

Using the rich analysis of Lesh et al. (2003) network model in the level of distribution of math representations and their transitions, which is categorized into four levels, level 1 is considered lower level of presentation (less than 0.20), level 2 is a low level (between (0.20- less than 0.40); level 3 is a medium level between (0.40-0.60); level 4 is a high level between (0.60 - less than 0.80) and very high is the level 5 (more than 0.80).

The results of the current study revealed -per Table 1- that there are about 374 representations in the unit, written symbols and verbal representations were in the medium level (38.24-38.50), whereas, real life situations and manipulative and pictures and diagrams were in the lower level (2.94-10.70).

Meanwhile, the results of the transitions of the representations showed that transition from the written symbol to the verbal representation has the highest percentage (29\%), followed by transition from verbal to written symbol representation with percentage (24\%) with a low level of distribution. Percentage of the transition from pictures and diagrams to written symbol representation is in the lower level(19\%), as the percentage of the transition from verbal to pictures and diagrams representation (9\%) and the percentages of the other transitions which were ranged between (1\%-4\%) very low. Table 2 shows these results.

The above mentioned revealed that the average of the transitions among the different representations was in the lower level, Figure 3 illustrates the percentages of the math textbook distribution of the multiple representations and the transitions among them. For instance, transition percentage from pictures and diagrams representation to manipulative representation was (4\%), while transitions percentage from manipulative representation to pictures and diagrams representation were $(2 \%)$. 


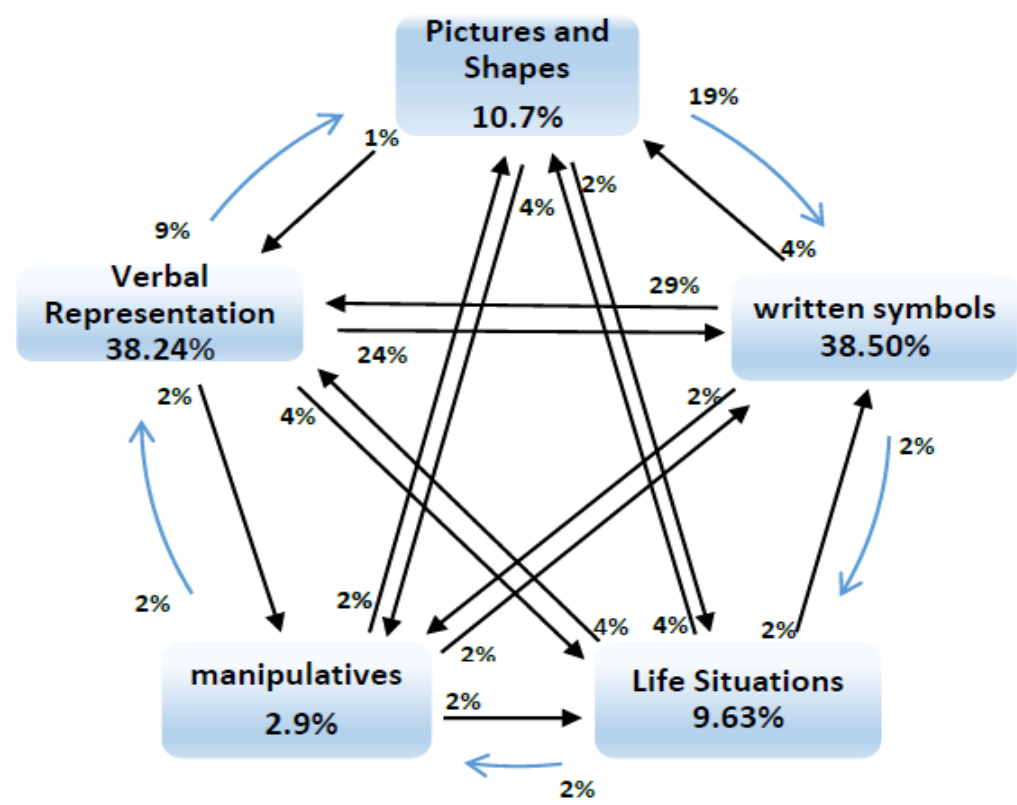

Figure 3. Percentages of the Math Textbook Distribution of the Representations and their Transitions

Despite, the results showed that the distributions of written symbols and verbal representations were in the medium level; the percentages of the of transitions were low, This reveals the fact that the multiple math representations were taught separately and not in a specific math content which affect students' understanding and learning and this align with the finding of Greeno and Hall (1997) who stated that teaching the multiple types of representations separately and not in a connected ways would not structure students' knowledge and engage them in meaningful and continuous ways. Juersivich, Garofalo and Fraser (2009) mentioned that the use of the representations as ends by themselves is not a functional in improving students' understanding.

Moreover, results of low representation by real life situations are in agreement with the results of the study of Yan and Lianghuo (2004) which cited that it is a common in most textbooks in the world the lack of real life situations representations such as the Unites States of America and China. Results of this study also align with the study of Bal (2015) that there is a lack in the use of representations in the curricula; and that the presentation of the curricula is still controlled by the traditional way.

The researcher revised the outlines of the Jordanian math curriculum and its objectives, where he found that the call of using the multiple representations such as tables, shapes, pictures and writing symbols. Other objectives were found which highlight the importance of math in the daily life, and acquiring math knowledge that helps the individual in his/her life. The results of the analysis showed a shortcoming in the math representations, as stated earlier, which the Ministry of Education in Jordan works to provide in the math curricula.

The researcher noted also that the concepts and activities are widely and repeatedly presented in the same representations; that there is an increase in the number of activities; and that this amount of activities burdens both the learners and teachers. This result is in agreement with those provided in the study of Yan and Lianghuo (2006) in the junior secondary stage schoolbooks in the United States of America. The researchers noted that the math books in the USA contain math problems much more than those in China, which burdens the learner.

In this concern, the researcher suggests decreasing the number of activities related to the same idea, and focus on presenting the idea or concept in more than one of these representations, especially real life situations. It is also suggested to use suitable transitions among these representations and encourage the students to justify their understandings,

In the light of these results, the researcher finds that one of the problems in math teaching-learning process is the shortage in the use of math representations, particularly real life situations, This view is in line with the study of Leinwand and Ginsburg (2007), who noted that the math textbooks in Singapore use multiple 
Table 3. The Percentages and Frequencies of teachers' use multiple representations of mathematics

\begin{tabular}{cccc}
\hline representations & frequencies & percentages & availability \\
\hline R & 130 & 42.48 & high \\
\hline L & 120 & 39.22 & medium \\
\hline H & 45 & 14.71 & low \\
\hline N & 4 & 1.31 & very low \\
\hline S & 5 & 1.63 & very low \\
\hline Total & 304 & & \\
\hline
\end{tabular}

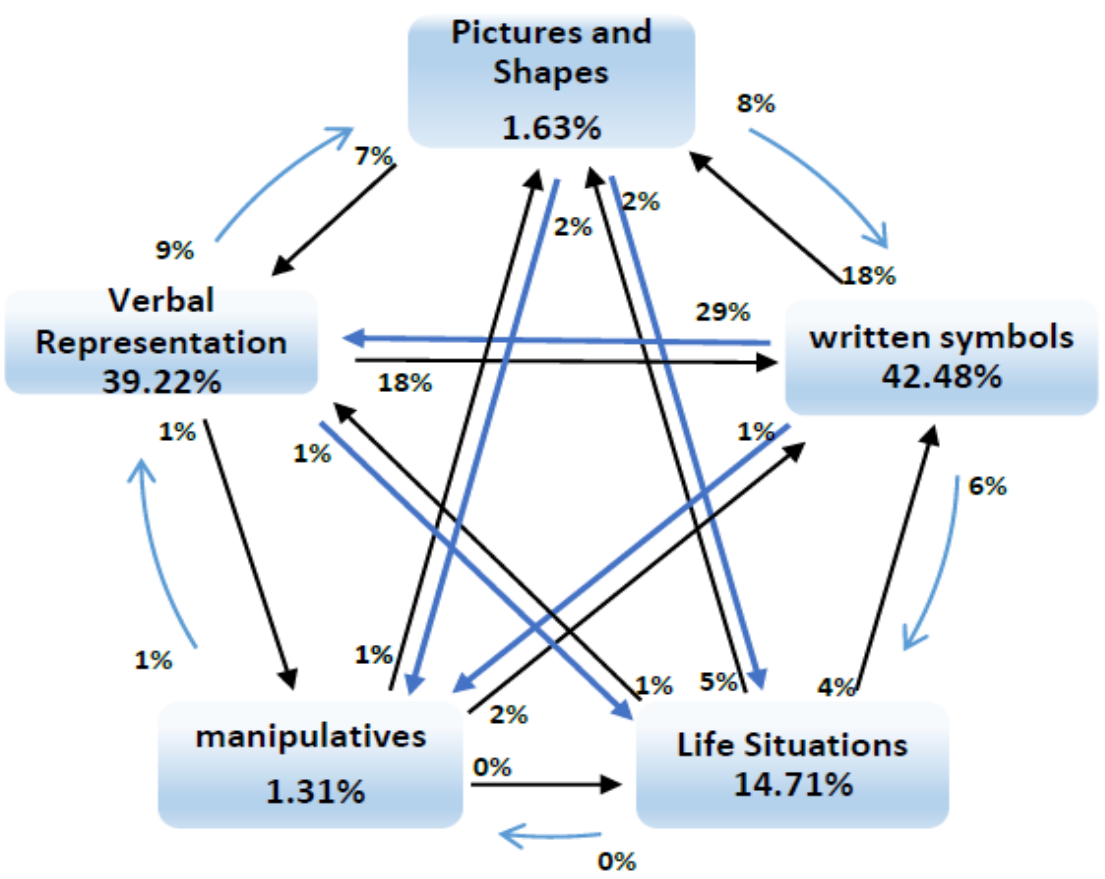

Figure 4. Percentages of Teachers' Use of the Representations and Transitions

math representations and transitions among them, to a wider scale, more than their use in the United States of America. In their point of view, this is the reason for the success of math curricula in Singapore.

Q2- To which extent teachers use representations and transitions among them while teaching the unit of Algebra in the textbook of $8^{\text {th }}$ grade?

Results of this question showed that the teachers' use of written symbols and verbal representations was very high; even so their use of the real-life situations representations was very low; it was much better presented than that of the math textbook. On the other hand, teachers' use of manipulative representations was very low, meanwhile the distribution of this representation in the textbook was better than teachers' use. The use of pictures and diagrams in the textbook was low; and the teachers' use was also very low, i.e. the textbook used them better than the teachers did. As for the transitions results, the use of both the book and teachers of the transitions was very low and low, respectively. Table 3 shows these results whereas Figure 4 shows the percentages of the teachers' use of the representations and their transitions.

The results indicated that the percentage of the use of written symbols representations by all teachers was (42.48\%), and verbal representations by all teachers was (39.22\%), which is the same distribution in the math textbook analysis. This means that the teachers used both representations at high and medium level as per Lesh et al. (2003) model. As for the real life situations representation, the percentage of teachers' use was $(14.71 \%)$, this indicates that their use of the real life situation representations is slightly better than that of the textbook, and that they were represented at very low level as per Lesh et al. (2003) model. The Percentage of their use of the manipulative representations was (1.31\%), which means that it was slightly less than that in the textbook, and that they were represented at very low level per Lesh et al. (2003) model. Finally, pictures 
and diagrams representations percentage was (1.63\%), indicating that their use of both representations was less than that of the textbook, and were represented at a low level as per the same model.

The results of the transitions distribution showed that the higher percentage of the use of transitions was within the written symbols and the verbal representations, meanwhile the teachers' use of this transitions was low (29\%), the teachers' use of these translation was the same on the textbook. The teachers' use of the transitions between the verbal to the written symbols representation was very low (18\%); and that teachers' use was less than that of the textbook.

For the teachers' use of the transitions between the written symbols and real life situations was (14.71\%) with very low level, which means that teachers' use of those transitions was better than the math textbook. On the other hand, teachers' use of the following transitions was less than on the textbook with a very low level (translations from written symbols to manipulative representation; from verbal to real life situations; from verbal to manipulative; from real life situations to manipulative; from manipulative to verbal; from manipulative to real life situations; from manipulative to pictures and diagrams; from pictures and shapes to symbols; from pictures and diagrams to verbal; and from pictures and diagrams to manipulative).

Meanwhile, teachers' use of the transitions among the representations (from written symbols to pictures and diagrams; from real life situations and written symbols; from real life situations to pictures and diagrams; and from pictures and diagrams to real life situations) was better presented than the textbook, but was represented at very low level as measured by Lesh et al. (2003) model. Finally, the average of teachers' use of the transitions from the verbal to the pictures and diagrams representations, and from manipulative to the written symbols representations was at the same rate of that in the textbook, and was represented at very low level according to Lesh et al. (2003) model.

The reason of the teachers' use of the real-life situations representation more than other types, they mentioned that they use real life situations to clarify certain concepts or avoid students' falling in mistakes. For instance," error of one student when he said: $12 \mathrm{~A}-4 \mathrm{~A}=8$. The teacher corrected the answer saying: if Khaled has 12 pieces of cookies, and he ate 4 pieces, "How many pieces of cookies remain with him? The student answered "8 pieces". Then the teacher said if we represent the cookie piece by A, then $12 \mathrm{~A}-4 \mathrm{~A}=8 \mathrm{~A}$."

The researcher noted that all participated teachers showed that the lack in the use of multiple math representations is due to lack of time to cover the given curriculum which contains a big amount of math activities (concepts, examples, class drills, exercise and problems). The results showed that teacher (5) was the less using the representations through real life situations, manipulative, and pictures and diagrams (very low level and almost near zero). He explained it "due to the lack of time and weakness of students, and focusing on concepts and examples". The researcher also noted that this teacher just use the material exited in the textbook. Meanwhile, teacher (1) used real life situations, manipulative, and pictures and diagrams representations, better than teacher (5) (at very low and low levels). Teacher (3) also used these representations at very low level, still better than teacher (5). Finally, teacher (5) used these transitions at lowest level comparing with the other sample participants, when investigating for reasons, one could find that teachers 5 has less years of experience in teaching 8th grade students.

\section{DISCUSSION AND CONCLUSION}

The current study employed the "content analysis" and "observation" as the method for interrupting the raw data in more significant meanings. The results indicated some main points: There is a lack of distribution of the multiple representations in the textbook moreover in the use of teachers while teaching, the findings are align with related research results such as (Treffert-Thomas, Viirman, Hernandez-Martinez \& Rogovchenko, 2017; You \& Quinn,2010) they found that there was a weakness in the distribution of the multiple representations, they also mentioned that the flexibility in the use of representations demonstrates the strengthen or weakness of the teachers' math knowledge and pedagogical content knowledge.

Some participated teachers mentioned that they didn't study any course related to multiple math representations during their university study, they hold BA degree in mathematics and didn't study any pedagogical course. It has been revealed the need to educate teachers on the use of the math representations and the transitions in the classrooms, You and Quinn (2010) cited that in teacher preparation there should be focus on the use of the multiple math representations and transitions among them in a harmonic manner, particularly representations by pictures and diagrams, real life situations, and written symbols, as well as transitions among them. 
The researcher found that the teacher's experience and knowledge affect their using and transitions of the multiple representations. Moreover, verbal representation was high, while its distribution on the math textbook and teachers' use of the other representations were low, and the use of math textbook and teachers for transitions among representations was low, and the concepts and activities are presented in a widespread use of the same representation, and there is overcrowding in activities, and that this amount of activities burdens the learners and teachers. In the light of the current study findings, the following suggestions could be put forward:

- The administration at the Ministry of Education in Jordan may benefit from the current study results concerning issues related to the curricula improvement and development.

- Researchers could use the tools of this study for future research.

- Comparisons could be conducted with other studies using content analysis approach.

- The current study may attract the attention of math teachers and supervisors to the importance of using the multiple representation and their transitions.

\section{Disclosure statement}

No potential conflict of interest was reported by the authors.

\section{Notes on contributors}

Mohammad Alkhateeb - Hashemite University, Jordan.

\section{REFERENCES}

Anthony, G., \& Walshaw, M. (2009). Characteristics of Effective Teaching of Mathematics: A View from the West. Journal of Mathematics Education, 2(2), 147-164.

Bal, A. (2015). Skills of Using and Transform Multiple Representations of the Prospective Teachers. Journal of Mathematical Behavior, 197(2015), 582-588. https://doi.org/10.1016/j.sbspro.2015.07.197

Bayazit, I. (2011). Prospective teachers' inclination to single representation and their development of the function concept. Educational Research and Reviews, 6(5), 436- 446.

Cai, J., \& Lester, F. (2005). Solution Representations and pedagogical representations in Chinese and U.S. classrooms. Journal of Mathematical Behavior, 24(3), 221-237. https://doi.org/10.1016/j.jmathb.2005. 09.003

Doerr, H., \& Lesh, R. (2011). Models and modelling perspectives on teaching and learning mathematics in the twenty-first century. In Trends in teaching and learning of mathematical modelling (pp. 247-268). Springer, Dordrecht. https://doi.org/10.1007/978-94-007-0910-2_26

Dreher, A., \& Kuntze, S. (2015). Teachers' professional knowledge and noticing: The case of multiple representations in the mathematics classroom. Educational Studies in Mathematics, 88(1), 89-114. https://doi.org/10.1007/s10649-014-9577-8

Dwi. R, Subanji, P, Hidayanto, E., \& Anwar, R. (2017). Process of Mathematical Representation Translation from Verbal into Graphic. Mathematic Education, 12(4), 367-381.

Gagatsis, A., \& Shiakalli, M. (2004). Ability to Translate from One Representation of the Concept of Function to Another and Mathematical Problem Solving. Educational Psychology, 24(5), 645-657. https://doi.org/10.1080/0144341042000262953

Greeno, J., \& Hall, B. (1997). Practicing Representation: Learning with and about Representational Forms. Phi Delta Kappa International, 78(5), 99-107.

Hwang, W., Chen, N., Dung, J., \& Yang, Y. (2007). Multiple Representation Skills and Creativity Effects on Mathematical Problem Solving using a Multimedia Whiteboard System. Educational Technology \& Society, 10(2), 191-212.

Juersivich, N., Garofalo, G., \& Fraser, V. (2009). Student Teachers' Use of Technology- Generated Representations: Exemplars and Rationales. Journal of Technology and Teacher Education, 17(2), 149173 
Koretsky, M., Keeler, J., Ivanovitch, J., \& Cao, Y.(2018), The role of pedagogical tools in active learning: a case for sense-making. International Journal of STEM Education, 5(18), 2-20. https://doi.org/10.1186/s40594-018-0116-5

Leinwand, S., \& Ginsburg, A. (2007). Learning from Singapore Math: The United States could benefit from looking at five elements driving the program's success. Educational Leadership, 65(3), 32-36.

Lesh, R, Post, T. \& Behr, M. (1987). Representations and translations among representations in mathematics learning and problem solving. Retrieved on 28/2/2018 from http://www.cehd.umn.edu/ rationalnumberproject/87_5.html

Lesh, R., \& Harel, G. (2003). Problem solving, modeling, and local conceptual development. Mathematical thinking and learning, 5(2-3), 157-189. https://doi.org/10.1080/10986065.2003.9679998

Lesh, R., \& Lehrer, R. (2003). Models and modeling perspectives on the development of students and teachers, $\begin{array}{llll}\text { Mathematical Thinking } \quad \text { Learning, } & \text { 5(2\&3), }\end{array}$ https://doi.org/10.1080/10986065.2003.9679996

Lesh, R., Cramer, K., Doerr, H., Post, T. \& Zawojewski, J. (2003). Using a translation model for curriculum development and classroom instruction. Retrieved on 5/1/2018, from: http://www.cehd.umn.edu/rationalnumberproject/03_1.html

Santulli, T. (2009). Representations from the Real World. Mathematics teaching in the middle school, 14(8), 466-473.

Treffert-Thomas, S., Viirman, O., Hernandez-Martinez, P., \& Rogovchenko, Y. (2017). Mathematics lecturers' views on the teaching of mathematical modelling. Education, 22(4), 121-145.

Tripathi, P. (2008). Developing mathematical understanding through multiple representations. Mathematics Teaching in Middle School. 13(89), 438-445.

Ulu, M. (2017). Examining the Mathematical Modeling Processes of Primary School 4th-Grade Students: Shopping Problem. Universal Journal of Educational Research, 5(4), 561-580. https://doi.org/10.13189/ujer.2017.050406

Yan, Z., \& Lianghuo, F. (2006). Focus on The Representation Of Problem Types In Intended Curriculum: A Comparison Of Selected Mathematics Textboks From Maibland China And The United States. International Journal of Science and Mathematics Education, 4(4), 609-626. https://doi.org/10.1007/s10763-006-9036-9

You, Z., \& Quinn, R. (2010). Prospective Elementary and Middle School Teachers' Knowledge of Linear Functions: A Quantitative Approach. Journal of Mathematics Education, 3(1), 66-76. 